Radial and Nonradial Pulsations as Probes of Stellar Physics

ASP Conference Series, Vol. 259, 2002

C. Aerts, T.R. Bedding, 8 J. Christensen-Dalsgaard, eds.

\title{
Interferometric-Doppler Imaging of Nonradial Stellar Pulsations
}

\author{
S. Jankov
}

Astronomical Observatory Belgrade, Yugoslavia

F. Vakili, A. Domiciano de Souza Jr.

Observatoire de la Côte d'Azur, Département FRESNEL, CNRS UMR 6528, France

E. Janot-Pacheco

Instituto Astronômico e Geofísico, Universidade de São Paulo, Brasil

\begin{abstract}
We present the imaging potential of a tomographic technique which combines time-resolved spectroscopy and long baseline interferometry, providing information that cannot be obtained otherwise with each of these techniques separately.
\end{abstract}

\section{Introduction}

The technique of Tomographic Imaging (Jankov \& Foing, 1992) is fully applicable to one of the most promising applications of Differential Speckle Interferometry (Beckers, 1982): the study of the surface of rapidly rotating stars with strong nonradial pulsations (Vakili \& Percheron, 1990). The applicability of this approach to the multi-mode pulsations observed in some stars (e.g. Jankov et al., 2000a) has been shown by Jankov et al. (2000b).

\section{Results}

Here we consider the example of mapping the brightness distribution due to a nonradial stellar pulsation mode with $m=4, \ell=5$ on a star tilted at $i=$ $45^{\circ}$, performing numerical experiments with realistic phase coverage, spectral resolution and signal-to-noise ratio expected for operating (the GI2T) or closeto-operating long baseline interferometers (VLTI, Keck).

A map recovered from spectra alone (classical Doppler Imaging) is shown on Fig. 1b, and should be compared to the original one (Fig. 1a) as well as to the maps obtained using single projection (parallel to rotation) of photocenter shifts (Fig. 1c) or spectra together with photocenter shifts parallel to rotation (Fig. 1d). Note the intrinsic limitations of Doppler Imaging on the reconstruction of features below the equator (loss of contrast), and spurious features introduced by high noise in measurements of photocenter shifts. 
a)

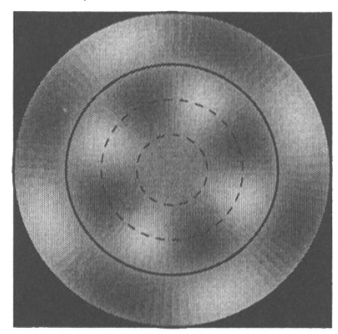

c)

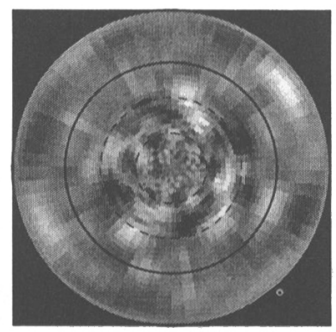

b)

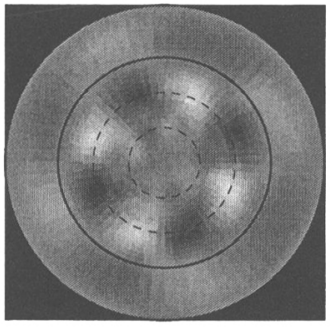

d)

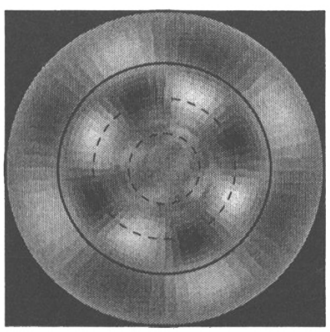

Figure 1. Pole-on projection of stellar surface brightness perturbation due to the $m=4, \ell=5$ mode on a star tilted at $i=45^{\circ}$ (a). The equator and the latitudes $30^{\circ}$ and $60^{\circ}$ are presented by full and dashed circles respectively. Maximum Entropy reconstructions from flux spectra (b), from photocenter shifts parallel to rotation (c) and from flux spectra together with photocenter shifts parallel to rotation (d).

A significant improvement with respect to previous reconstructions has been obtained using both spectra and photocenter shifts parallel to rotation.

\section{Conclusion}

We have shown that accurate maps of stellar nonradial pulsations can be obtained using the Interferometric-Doppler Imaging approach and regularized inversion with the Maximum Entropy Method.

\section{References}

Beckers, J.M. 1982, Opt. Acta, 29, 361

Jankov, S., Janot-Pacheco, E., \& Leister, N.V. 2000a, ApJ, 540, 535

Jankov, S., Janot-Pacheco, E., \& Leister, N.V. 2000b, in ASP Conf. Ser., Vol. 214, The Be phenomenon in early-type stars, eds. M. Smith, H. Henrichs, \& J. Fabregat, (San Francisco ASP), 264

Jankov, S. \& Foing, B. 1992, A\&A, 256, 533

Vakili, F. \& Percheron I. 1990, in Rapid Variability of OB Stars: Nature and Diagnostic Value, ed. D. Baade, 77 


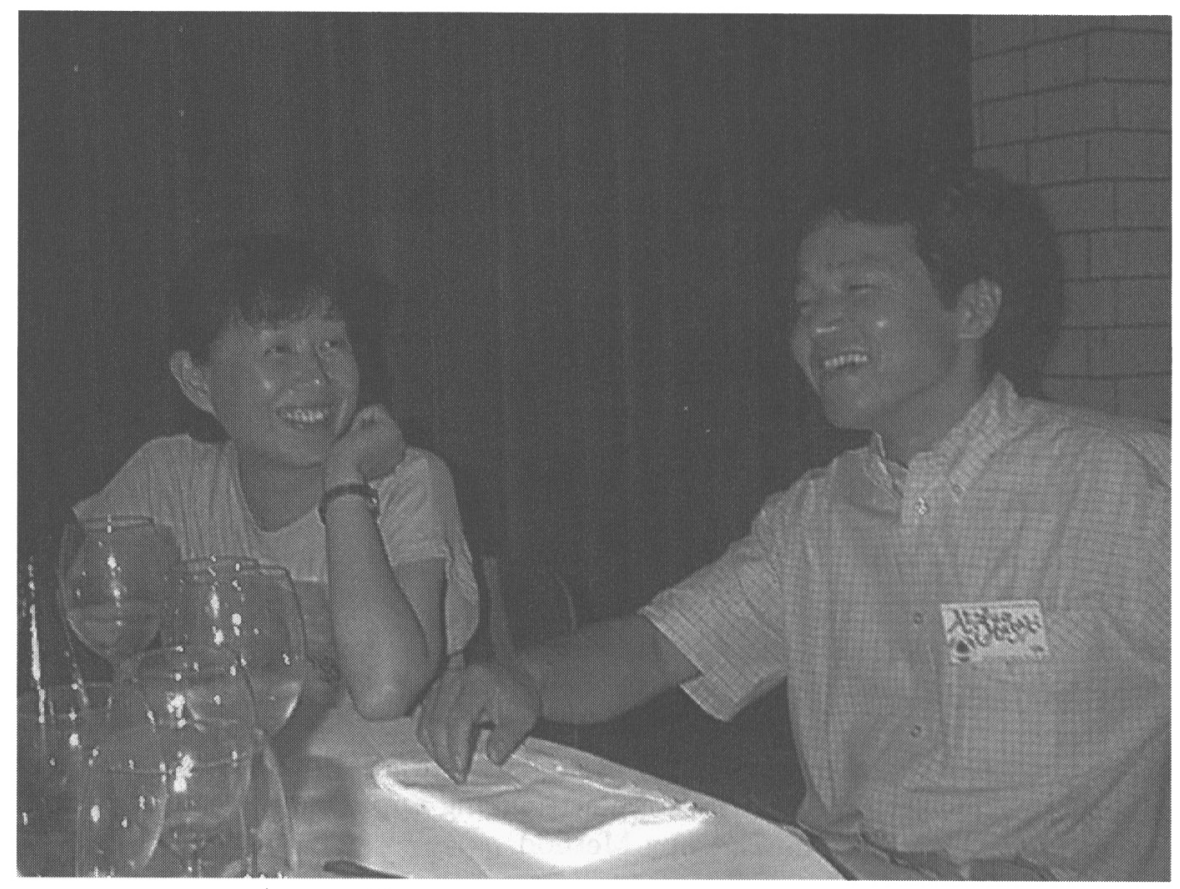

Atsuo Okazaki and his wife having great fun. 